OPEN ACCESS

Edited by:

Offer Erez,

Soroka Medical Center, Israel

Reviewed by:

Sinno Simons,

Erasmus Medical Center, Netherlands

Li Wang,

University-Town Hospital of Chongqing Medical University, China

${ }^{*}$ Correspondence:

Xin Luo

14802315@qq.com

Hongbo Q

qihongbocy@gmail.com

tThese authors have contributed equally to this work

Specialty section

This article was submitted to

Neonatology,

a section of the journal

Frontiers in Pediatrics

Received: 01 March 2021

Accepted: 16 August 2021 Published: 07 September 2021

Citation:

Huang D, Liu Z, Liu X, Bai Y, Wu M,

Luo X and Qi H (2021) Stress and Metabolomics for Prediction of

Spontaneous Preterm Birth: A

Prospective Nested Case-Control

Study in a Tertiary Hospital.

Front. Pediatr. 9:670382.

doi: 10.3389/fped.2021.670382

\section{Stress and Metabolomics for Prediction of Spontaneous Preterm Birth: A Prospective Nested Case-Control Study in a Tertiary Hospital}

\author{
Dongni Huang ${ }^{1,2,3,4 t}$, Zheng Liu 1,2,3†, Xiyao Liu ${ }^{1,2,3}$, Yuxiang Bai ${ }^{1,2,3}$, Mengshi Wu ${ }^{1,2,3}$, \\ Xin Luo ${ }^{1,2,3 *}$ and Hongbo Qi ${ }^{1,2,3 *}$ \\ ' Department of Obstetrics, The First Affiliated Hospital of Chongqing Medical University, Chongqing, China, \\ ${ }^{2}$ China-Canada-New Zealand Joint Laboratory of Maternal and Fetal Medicine, Chongqing Medical University, Chongqing, \\ China, ${ }^{3}$ Joint International Research Laboratory of Reproduction and Development of Chinese Ministry of Education, \\ Chongqing Medical University, Chongqing, China, ${ }^{4}$ Department of Obstetrics, Chongqing Health Center for Women and \\ Children, Chongqing, China
}

Spontaneous preterm birth (sPTB) is the leading cause of infant morbidity and mortality worldwide. Deficiency of effective predict methods is an urgent problem that needs to be solved. Numbers of researchers spare no efforts to investigate differential indicators. To evaluate the value of the differential indicators, a prospective nested case-control study was carried out. Among an overall cohort of 1,050 pregnancies, 20 sPTB pregnancies, and 20 full-term pregnancies were enrolled in this study. Participants were followed-up until labor. The psychological profile was evaluated utilizing the Zung Self-Rating Depression Scale at 11-14 weeks. Stress-related biomarker-cortisol and metabolites were detected by Electrochemiluminescence Immunoassay (ECLIA) and Gas Chromatography-Mass Spectrometry (GC-MS) in serum samples during pregnancy, respectively. The expression level of cortisol was up-regulated in serum and the score of the Zung Self-Rating Depression Scale was significantly higher in the SPTB group when compared to the control group. Note that, 29 metabolomics were differentially expressed between the SPTB group and the control group. The scores of the Zung Self-Rating Depression Scale, the level of cortisol, Eicosane, methyltetradecanoate, and stearic acid in serum were selected to establish the model with lasso logistic regression. Validation of the model yielded an optimum corrected AUC value of $89.5 \%, 95 \% \mathrm{Cl}: 0.8006-0.9889$ with a sensitivity of $100.0 \%$, and specificity of $78.9 \%$. In conclusion, this study establishes a prediction model of SPTB with five variables, which may predict SPTB more accurately and sensitively in the second trimester.

Keywords: spontaneous preterm birth, cortisol, metabolomics, predictive model, serum 


\section{INTRODUCTION}

Spontaneous preterm birth (sPTB), defined as delivery before 37 weeks of gestation, is a prevalent complication of pregnancy. About 15 million preterm infants born each year, accounting for $\sim 10.6 \%$ of all live births worldwide, and the incidence of sPTB in China between 2015 and 2016 was $7.3 \%(1,2)$. Furthermore, those preterm neonates who do survive have higher rates of short-term and long-term morbidities. Comparing with full-term infants, preterm infants may have higher incidences of some common complications, including respiratory distress syndrome, feeding difficulties, hypoxic ischemic encephalopathy, and developmental disabilities (3-5). In addition, threatened preterm delivery increases the possibility of final preterm delivery, and there are no clinical symptoms and signs in the early stage and lacks of accurate and specific predictive indicators in clinical practice. Successful identification of at-risk women at an early stage will be a major leap forward in the discovery science of preterm birth by allowing identification of the most informative population for pathophysiology understanding and intervention development. Therefore, there is an urgent need to identify other potential factors involved in SPTB to establish a new model for prediction.

sPTB has been linked to a complex cluster of overlapping biomedical, social and psychological factors (6). Among them, stress is widely considered as a contributor of preterm birth. Some studies have shown that stress, as indicated by stressful life events, perceived stress, and depression, is higher among women who go on to deliver preterm. Furthermore, stress can be triggered from many different sources, including low socioeconomic status, elderly age, and social support (7). The maturation of hypothalamic-pituitary-adrenal axis (HPA) axis can create a pre-control hormone environment for the myometrium and enhance uterine contraction. In addition, glucocorticoid induces the expression of related enzymes in placenta, which converts progesterone into estrogen. Cortisol, the hormonal endpoint of physiologic stress-related activation of the hypothalamic-pituitary-adrenal axis, has been associated with fetal matureness and parturition by stimulating prostaglandin secretion and promoting uterine contraction, and is often cited as a potential mediator between stress and pregnancy outcomes. Cortisol fluctuates rapidly in response to even subtle changes in the environment and single serum measurement do not reflect chronic levels (8). Additionally, previous studies carried out in PTB women found that high cortisol levels were a reliable predictor for preterm birth at $48 \mathrm{~h}$, but the predictive index is dull and the accuracy is not high (9). Hence, it is necessary to combine multiple factors to improve the accuracy and efficiency of prediction.

Metabolomics, the investigation of low molecular weight biochemical (metabolites) in cells, tissues, or organisms, which developed from genomics, transcriptomics, and proteomics, is a comprehensive analysis of metabolites investigated to search for disease-specific metabolic signals, which could be used as biomarkers $(10,11)$. Metabolomics has previously been successfully utilized in the diagnosis of pregnancy complications, such as preeclampsia and fetal growth restriction (12).
Mass-spectrometry-based metabolic profiling is increasingly used to uncover new biomarkers for diagnosis, prognosis, pathogenesis clarification and potential therapeutic targets for clinical treatment $(13,14)$. Thus, metabolomics is a powerful tool to find biomarkers. Given this, in this study, we combine stress biomarkers and metabolites to establish a predictive model for sPTB, which might have a crucial effect on preterm birth treatment.

\section{MATERIALS AND METHODS}

\section{Sample Collection}

Participants enrolled in the nested case-control study were selected from a prospective cohort. All enrolled pregnancies were followed up from enrollment to 42 days after birth, and their basic and clinical information were collected. This study began recruiting participants in April 2017. If the number of losing follow-up was more than expected, more pregnancies were recruited. The ethics approval was obtained from the Ethics Committee of the First Affiliated Hospital of Chongqing Medical University. All the participants signed informed consent and were enrolled into the study if they were a singleton pregnancy. Then they should be 10-14 weeks of pregnancy, Depressive symptoms of participants were assessed by the Zung Self-Rating Depression Scale (ZSDS) in the first trimester (10-14 gestational weeks). ZSDS $\geq 50$ was used as the cut-off score for the presence of depressive symptoms. Bio-samples (serum) were collected at each visiting point. sPTB was defined according to the criteria published by the American College of Obstetrics and Gynecology. All the pregnancies with chronic medical disorders were excluded, including gestation diabetes mellitus, cardiovascular disease, chronic renal disease, collagen disorders, chronic hypertension, and metabolic diseases. Finally, $20 \mathrm{sPTB}$ pregnancies $(n=20)$ and 20 full-term pregnancies $(n=20)$ were enrolled in our study (Figure 1). The samples were obtained from 8:00 a.m. to 10:00 a.m. in the hospital. After obtaining the samples, they were transferred to the laboratory with ice boxes within $1 \mathrm{~h}$ and centrifuged at 3,000 rpm for $10 \mathrm{~min}$ at $4^{\circ} \mathrm{C}$. Serum samples were kept at $-80^{\circ} \mathrm{C}$ until analysis.

\section{Laboratory Analysis}

Serum cortisol levels were detected by a single stage electrochemiluminescence immunoassay (ECLIA, Cobas 8000 Roche Diagnostics Scandinavia AB). The method had a coefficient of variation (CV) of $7 \%$ at $100 \mathrm{nmol} / \mathrm{L}, 5 \%$ at 570 $\mathrm{nmol} / \mathrm{L}$, and $5 \%$ at $990 \mathrm{nmol} / \mathrm{L}$.

\section{Sample Preparation and Analysis by GC-MS}

To determine the practicability of using the maternal serum metabolome in identifying the asymptomatic women who were at risk of sPTB in the second trimester, GC-MS was performed. Serum samples were thawed and analyzed by GC-MS according to the method described by Li et al. (10). A $100 \mu \mathrm{L}$ aliquot of serum was centrifuged at $15,000 \mathrm{rpm}$ for $10 \mathrm{~min}$ at $4^{\circ} \mathrm{C}$. Then, the mixture was obtained by spiking an internal standard (2chlorophenylalanine, $0.3 \mathrm{mg} / \mathrm{mL}$ ) into serum and then vortexed 
Inclusion criteria : 1, singleton pregnant women, 2, 11-14 weeks of pregnancy, 3 , no previous mental illness, 4 , no previous major underlying diseases, 5 , no pregnancy with internal and surgical diseases

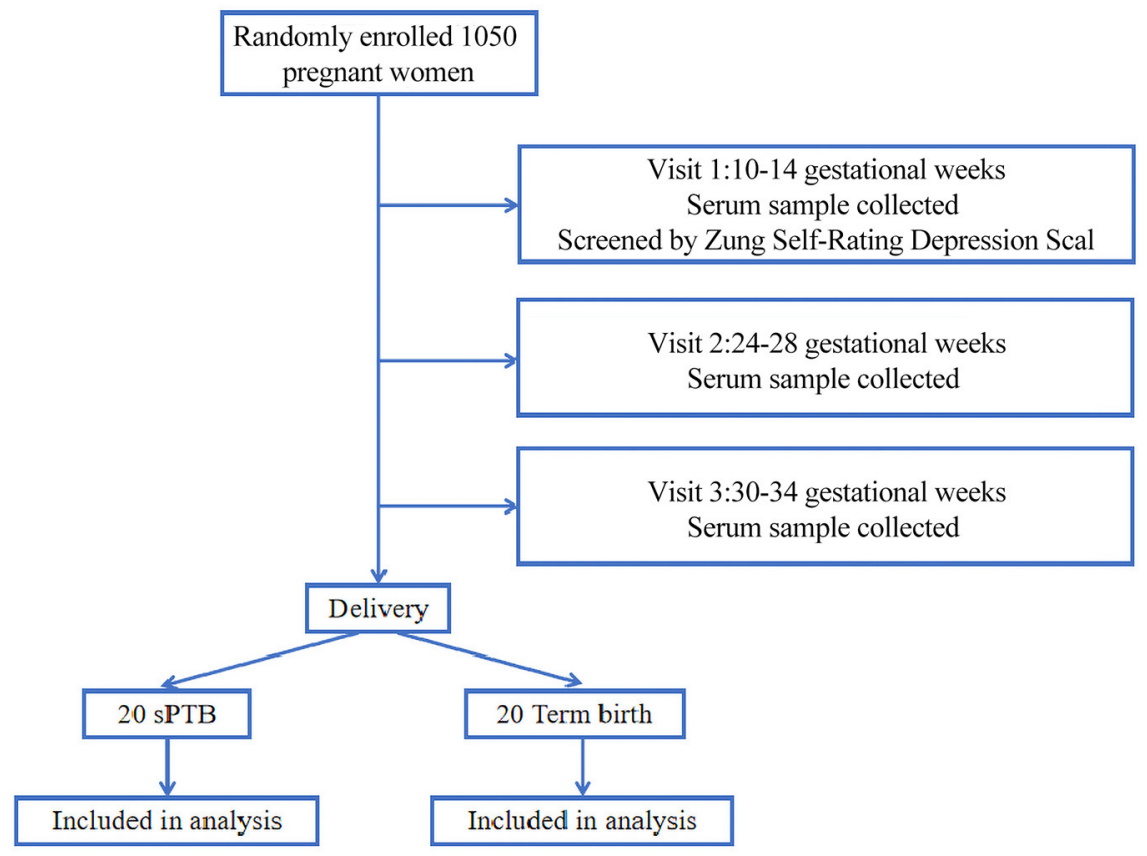

FIGURE 1 | The flow chart of our cohort.

for $10 \mathrm{~s}$. This mixture was then extracted with $150 \mu \mathrm{L}$ of methanol-acetonitrile and vortexed for $30 \mathrm{~s}$, followed by cooling for $10 \mathrm{~min}$ at $-20^{\circ} \mathrm{C}$ and then centrifuging at $15,000 \mathrm{rpm}$ for $10 \mathrm{~min}$ at $4^{\circ} \mathrm{C}$. An aliquot of the $150 \mu \mathrm{L}$ supernatant was placed in a glass sampling vial and dried under vacuum at room temperature. The residue was first reconstituted in $80 \mu \mathrm{L}$ of methoxyamine $(15 \mathrm{mg} / \mathrm{mL}$ in pyridine), then vortexed for $30 \mathrm{~s}$, and kept at $37^{\circ} \mathrm{C}$ for $90 \mathrm{~min}$. Finally, $80 \mu \mathrm{L}$ of bis(trimethylsilyl) trifluoroacetamide (BSTFA) (1\% trimethylchlorosilane) and 20 $\mu \mathrm{L}$ of $\mathrm{n}$-hexane were added, and the extract was kept at $70^{\circ} \mathrm{C}$ for $60 \mathrm{~min}$. Derivatized extract was separated by a GC7890 chromatography system using a ZB-1701 GC capillary column $(30 \mathrm{~m} \times 250 \mu \mathrm{m}$ id $\times 0.15 \mu \mathrm{m}$ with a $5 \mathrm{~m}$ guard column, Phenomenex) and analyzed by a MSD5975 mass spectrometry (Agilent, California, USA) with electron impact ionization via electron emission at $70 \mathrm{eV}$. The GC temperature program and MS parameters were set according to the protocol described in Smart et al. (15).

\section{Metabolites Validation, Data Mining, and Statistical Analysis}

Automated Mass Spectral Deconvolution \& Identification System software was employed for metabolite deconvolution. The compounds were identified by comparing the MS fragmentation patterns and respective GC retention time to an in-house MS library established using chemical standards. The remaining putative compounds were identified using a commercial NIST mass spectral library. The MassOmics XCMS R-based script was used to extract the relative concentration of the metabolites through the peak height of the most abundant fragmented ion mass. To improve quantitative robustness and minimize human and instrumental variabilities, the relative abundances of the identified compounds were normalized in the order of multiple internal standards, median centeringbatch correction via QC samples. Student's $t$-test, non-parametric Mann-Whitney $U$ test, Chi-square test, and Fisher's exact test were performed in $\mathrm{R}$ to compare clinical characteristics between the control and the sPTB group. Before metabolomic statistical analysis, the serum metabolite profiles were adjusted to Gaussian distribution through log transformation and Pareto scaling. Partial least squares discriminant analysis (PLSDA) was performed to compare serum metabolome profiles between two groups using the Metaboanalyst 3.0 package for $\mathrm{R}(16)$.

\section{Predictive Model Establishment Data Standardization}

In the original data, each variable was continuous. However, the data were various and differed in units. Hence, the model parameter estimation coefficients were standardized before analysis in order to overcome the influence of the dimension and make it comparable. 
TABLE 1 | Medians or Counts (\%) for demographic and clinical variables.

\begin{tabular}{|c|c|c|}
\hline Variables & Term birth $(n=20)$ & $\begin{array}{l}\text { Spontaneous } \\
\text { preterm } \\
\text { birth }(n=20)\end{array}$ \\
\hline Maternal age $\left(\right.$ years) ${ }^{\star}$ & $33.10 \pm 1.275$ & $32.50 \pm 1.222$ \\
\hline Maternal BMl* & $25.94 \pm 0.6721$ & $27.13 \pm 0.6119$ \\
\hline ZUNG score ${ }^{\#}$ & $42.56 \pm 1.123$ & $49.19 \pm 0.6494$ \\
\hline Birth weight $(g)^{\#}$ & $3381 \pm 66.21$ & $2619 \pm 116.1$ \\
\hline \multicolumn{3}{|l|}{ Birth gender } \\
\hline Boys & $9(45 \%)$ & $8(40 \%)$ \\
\hline Girls & $11(55 \%)$ & $12(60 \%)$ \\
\hline \multicolumn{3}{|c|}{ Gestational age at delivery (weeks) } \\
\hline$<28$ & N/A & $0(0 \%)$ \\
\hline 28-32 & & $1(5 \%)$ \\
\hline 32-34 & & $3(15 \%)$ \\
\hline $34-37$ & & $16(80 \%)$ \\
\hline \multicolumn{3}{|c|}{ Preterm premature rupture of membranes } \\
\hline Yes & N/A & $10(50 \%)$ \\
\hline No & & $10(50 \%)$ \\
\hline
\end{tabular}

BMI, body mass index.

Data are presented as mean $\pm S D$.

${ }^{*}$ t-test $P$-value 0.7359 for age, 0.1977 for BMI, respectively.

${ }^{\#} t$-test $P$-value $<0.0001$ for ZUNG Score and birth weight.

a. Lasso logistic regression: the glmnet package of $\mathrm{R}$ software (version 3.6.1) was employed to implement Lasso logistic regression, and the value of $\lambda$ was determined through crossvalidation.

b. Only Lasso was used for feature selection, and then a supporting vector machine prediction model was established using radial basis function (RBF), polynomial, and Sigmoid kernel function. The independent prediction ability of the model was tested by Leave-One-Out method (LOO): Take one sample at a time from the $\mathrm{n}$ samples as the test set, and use the remaining $n$ - 1 samples to form the training set.

\section{RESULTS}

\section{Participants}

The differences of demographic and clinical characteristics between both groups were presented in Table 1. This information revealed that both groups had similar BMI and age. The difference between the two groups was not statistically significant. Additionally, the association between the lower birth weight infants and the SPTB pregnancies was found. Participants complicated with sPTB had lower birth weight infants and higher ZUNG scores $(P<0.001)$, which indicated that higher ZUNG scores was a potential clinical predictor for sPTB.

\section{Expression of Cortisol in Serum Samples During Pregnancy}

Firstly, the expression level of cortisol was detected in serum samples. According to our results, the expression level was found to increase from the first trimester to the third trimester. After we analyzed the data, the association between sPTB and

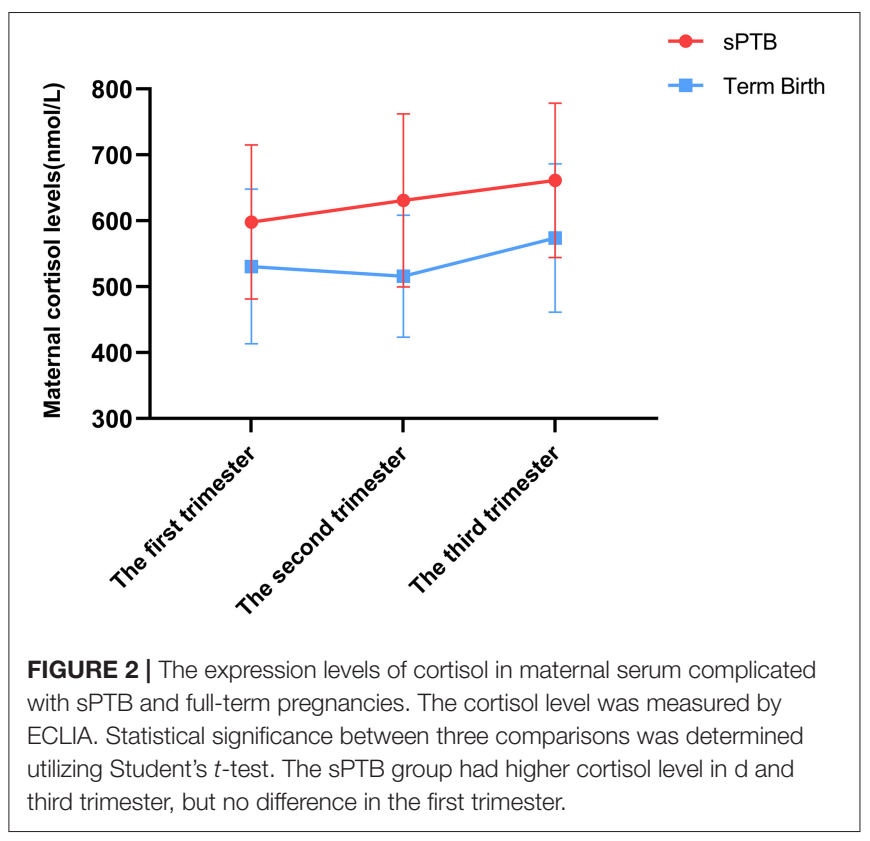

maternal cortisol levels across pregnancy was detected. In the first trimester, there was no significant difference in cortisol levels between both groups (Term vs. $\mathrm{PPTB}, P=0.081$ ). However, in the second trimester, cortisol levels of serum in the sPTB group were detected dramatically higher than those in the full-term group (Term vs. sPTB, $P=0.005$ ). Moreover, such differences still persisted in the third trimester (Term vs. $\mathrm{PPTB}, P=0.043$ ) (Figure 2).

\section{Metabolites Significantly Associated With sPTB}

A total of 119 compounds were identified from the in-house MCF spectral library and NIST library (https://www.nist.gov/nistresearch-library). The partial least squares discriminant analysis (PLS-DA) showed the most distinct separations between the sPTB group and the full-term group (Figures 3A,B). As shown in Figure 3C, 29 serum metabolites were detected significantly different in concentrations between the two groups with $p$-value and $q$-value $<0.05$ and 0.05 , respectively. Among them, 18 metabolites were validated significantly upregulated in the sPTB group includes 4 alkanes, 7 amino acid derivatives, 1 glycolytic intermediate, and 6 prganic acids. Conversely, 11 metabolites were found to be significantly downregulated in the SPTB group includes 4 long-chain saturated fatty acids, 1 Medium-chain saturated fatty acid, 1 TCA cycle intermediate, 4 long-chain unsaturated fatty acids, and 1 branch Unsaturated fatty acid.

\section{Construction and Validation of the Prediction Model for SPTB}

To further shortlist the significant metabolites that might have clinical implications, Lasso was carried out for all metabolites. The results showed that when $\lambda$ min $=0.07439764$, that was, $\log \left(\lambda_{\min }\right)=-2.598331$ (left dotted line in Figure 4A), the 
A

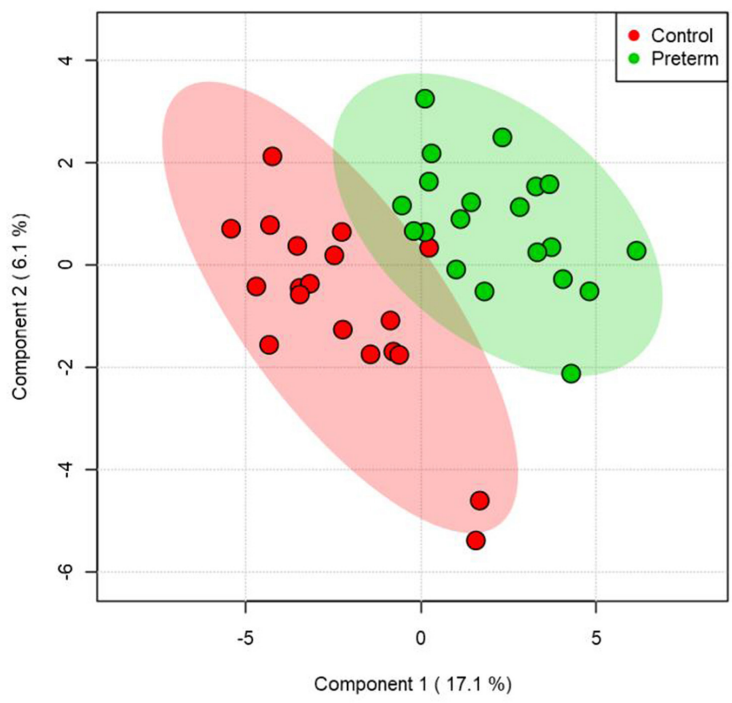

B

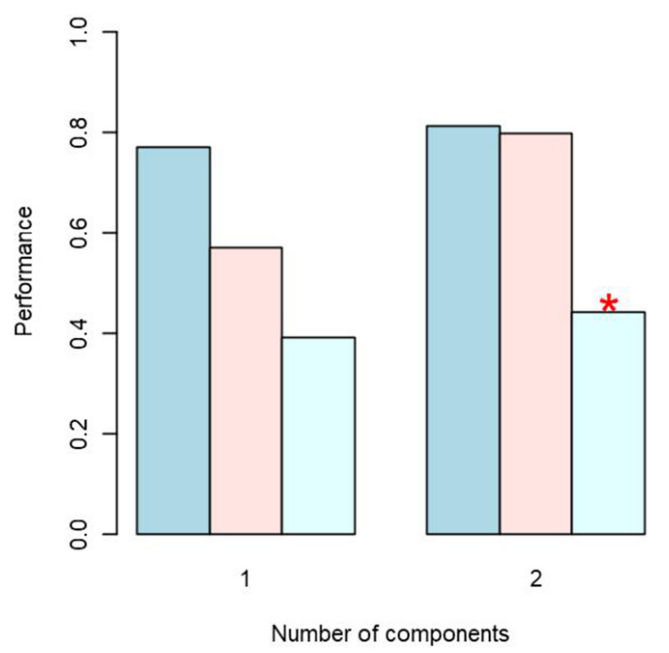

$\square$ Accurac

$\square$ R2

$\square$ Q2

C
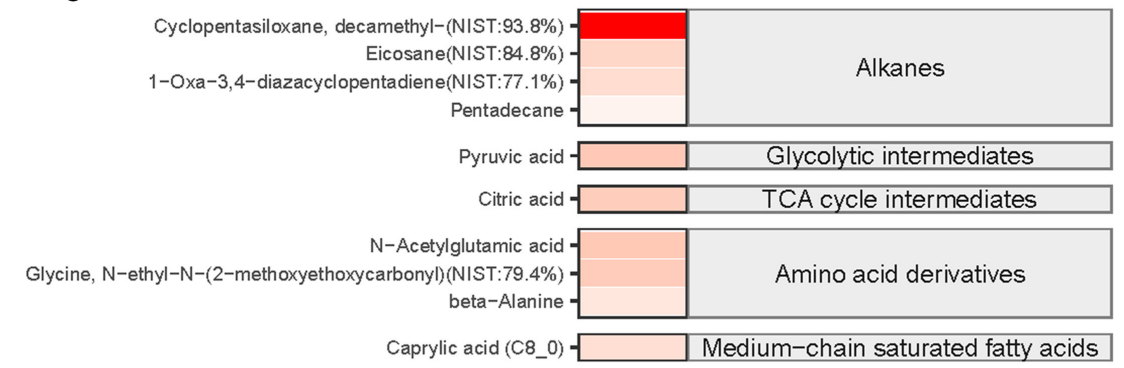

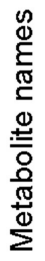

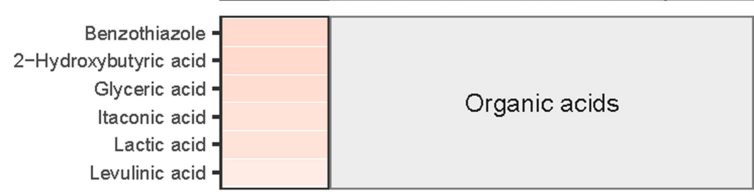

$\log 2$ Scale

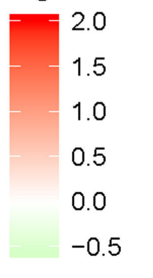

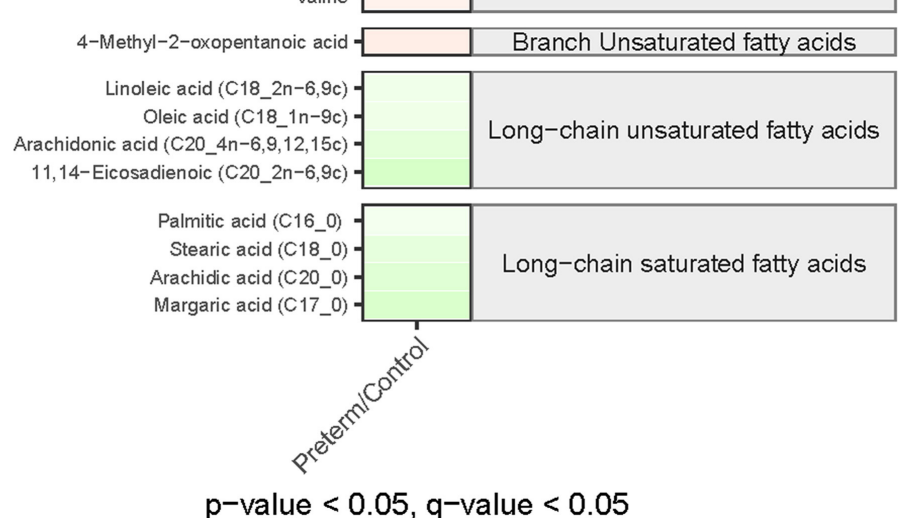

FIGURE 3 | Partial least squares discriminant analysis (PLS-DA) and the heatmap of the serum metabolome between the sPTB group and the full-term group. (A,B) PLS-DA of the serum metabolome between the sPTB group and the full-term group, including a measure of prediction model performance. (C) The heatmap shows the differentially expressed metabolites. The relative concentrations of serum metabolites were demonstrated through log 2 scale. The red color blocks indicate a higher expression level, and the green color blocks indicate a lower expression level. Only the metabolites with a $p$-value of $<0.05$ and a $q$-value of $<0.05$ were displayed. 

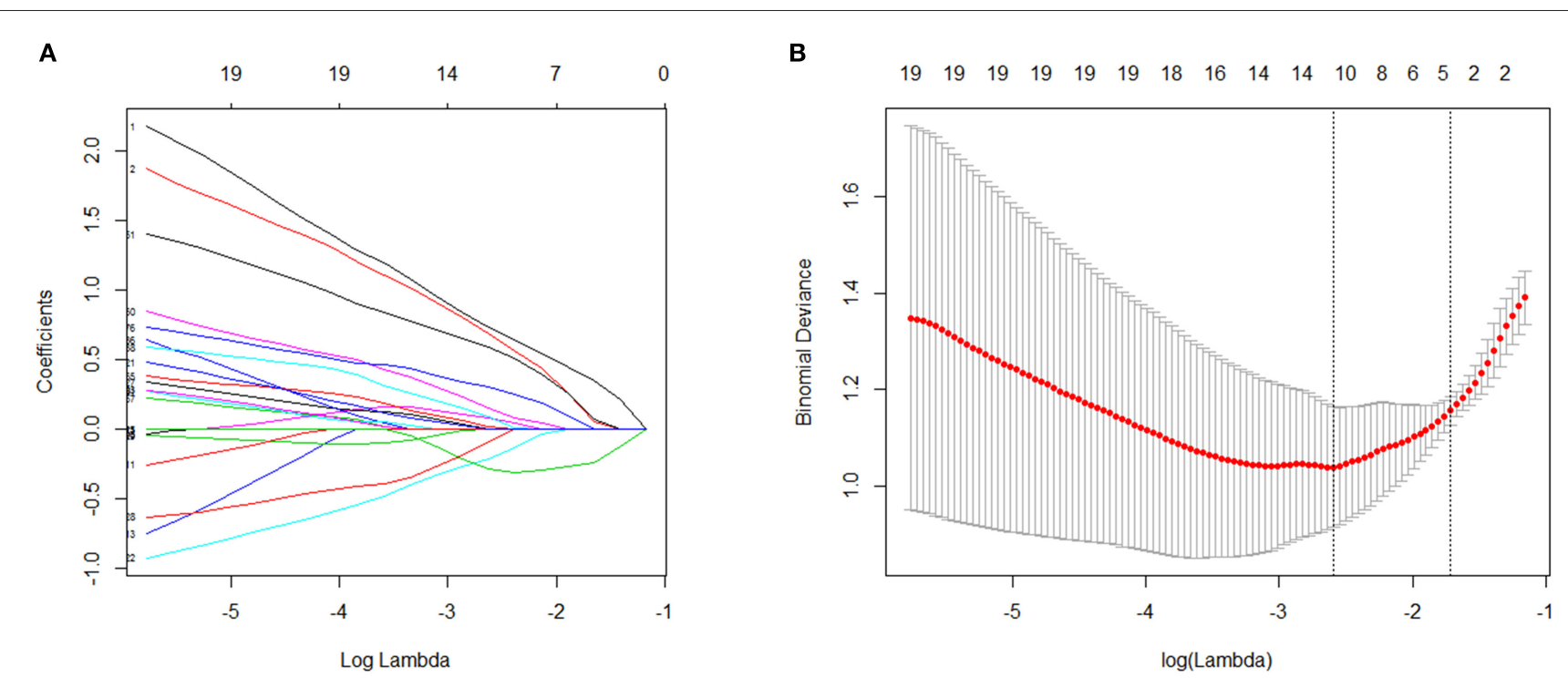

FIGURE 4 | Validation screened by Lasso. (A) The changes of coefficients with parameters were plotted in Lasso regression. (B) The change diagram of binomial device with parameter $\ln \lambda$ was drawn through 3-folds cross validation.

binomial device reached the minimum value. In addition, $\lambda$ 1 se $=0.1800518$, that was, $\log \left(\lambda_{1 \mathrm{se}}\right)=-1.714511$ (dotted line on the right side in Figure 4B) was the best $\lambda$ value corresponding to the simplest model within a standard error of $\lambda_{\text {min }}$. Finally, the coefficient of the selected model was $\lambda_{1 \text { se }}$, corresponding to five variables selected to enter the regression model, namely ZUNG score, serum cortisol level, Eicosane level, i-Propyl 12-methyltetradecanoate level, and Stearic acid level. If variable coefficient was a negative number, indicating the negative correlation between the function and the variable, that means the probability of premature delivery risk decreased with the increase of the index value; otherwise, the greater the index value, the higher premature delivery risks (Table 2). Validation of the model yielded an optimum corrected AUC value of $89.5 \%, 95 \%$ CI $(0.8006-0.9889)$ with a sensitivity of $100.0 \%$ and specificity of $78.9 \%$. The model's independent prediction ability was tested by LOO after establishing the model using RBF, polynomial, and Sigmoid kernel function with the accuracy of 94.9, 89.7, and 97.4\%, respectively, as illustrated in Table 3.

\section{DISCUSSION}

To the best of our knowledge, this is the first prospective nested case-control study to investigate whether psychosocial factors and metabolites can predict spontaneous preterm birth in the second trimester. In this study, we found that concentrations of serum cortisol and scores of ZUNG depression scale were positively associated with the risk of sPTB. Moreover, 29 aliphatic acids, expressed differentially between the sPTB and the full-term maternal serum were identified through GC-MS in this study. The strengths of this study lie in the prospective design and the rapid determination of sPTB risk in the second
TABLE 2 | Standardized regression coefficient of variables.

\begin{tabular}{lc}
\hline Variables & Standardized regression coefficient \\
\hline Intercept & 0.04908751 \\
ZUNG score & 0.37593216 \\
Cortisol level (intercept) & 0.10916823 \\
i-Propyl 12-methyltetradecanoate level & 0.02658327 \\
Eicosane level & 0.11962625 \\
Stearic acid level & -0.24982455
\end{tabular}

TABLE 3 | Test the model's independent prediction ability by LOO.

\begin{tabular}{lcc}
\hline Function & Correct number & Accuracy \\
\hline RBF & 37 & $94.90 \%$ \\
Poly & 35 & $89.70 \%$ \\
Sigmoid & 38 & $97.40 \%$
\end{tabular}

trimester. Besides, validation of the model yields high sensitivity and specificity.

Preterm birth, as a multifactor syndrome, can be triggered by stress (17). Women with likely diagnosis of a major depressive episode are at a 4 -fold increased risk of preterm birth (18). Previous studies have found that cortisol levels appeared higher in sPTB compared to term birth. Hence, the high levels of stress biomarker-cortisol may play an important role in the prediction of preterm birth. Additionally, the twin pregnancies who experienced social stress also undergone preterm birth (19). Cortisol, a biomarker for indication of delivery, are capable of being refined by models that incorporate in other biomarkers. However, few predictive models can predict preterm birth in 
mid-pregnancy by assessing maternal stress and metabolites. Grasso Lucia et al. found that serum cortisol was more sensitive and specific than urine cortisol throughout pregnancy (20). Therefore, serum cortisol was included in this model to ensure the accuracy and reliability of the preliminary model. To explore the relationship between pregnancy complications and prenatal exposure factors, a large pregnancy cohort was established. Therefore, the serum cortisol and metabolic concentrations observed in our study can be considered representative of the general maternal level at some scale. In addition, this prospective study can provide first-hand information of stress and spontaneous preterm birth directly with little bias.

Few studies have applied metabolomic techniques to understand sPTB in asymptomatic pregnant women. A total of 29 aliphatic acids were differentially expressed between the sPTB and the full-term maternal serum in the second trimester by GC-MS. Among them, we further enlisted i-Propyl 12-methyltetradecanoate, Eicosane, and Stearic acid into our model for sPTB predicting to improve its performance over a model with cortisol alone (21). Long chain fatty acids are needed for the development of brain, retina, and nervous system of fetal during the third trimester of pregnancy. Stearic acid (a long chain saturated fatty acid) level was found lower in the preterm birth infants than in the term birth infants (22). Actually, there are very few studies indicating the direct relationship between i-Propyl 12-methyltetradecanoate, Eicosane and preterm birth. Fortunately, Eicosane can be found in tobacco (23), previous studies also have found that Mother quit smoking can effectively reduce the risk of recurrent preterm birth (24). In addition, oxidative stress may lead to degradation of cell membranes by lipid peroxidation, following by conversion of polyunsaturated fatty acids to volatile alkanes. Moreover, the relationship of oxidative stress-associated process and $\mathrm{SPTB}$ have also been previously reported. These may indicate why an abnormal increase in Eicosane may predict preterm birth. However, investigation of the potential relationship of i-Propyl 12-methyltetradecanoate and preterm birth is further required.

Though a variety of predictive models of preterm birth have been developed, the inability to meet both sensitivity and specificity in mid-pregnancy is a common problem in current preterm prediction models. For instance, a prediction model of spontaneous delivery within 7 days was developed through the diagnosis of amniotic cavity and/or intra-amniotic inflammation. The models presented a high diagnostic performance, showing an area under curve (AUROC) of 0.86 [95\% confidence interval (CI) $0.77-0.95$ ] with a detection rate of spontaneous delivery within 7 days of $87 \%$, a false-positive rate of $33 \%$, negative predictive value of $80 \%$, and negative Likelihood ratio of 0.1908 (25). However, it is an invasive diagnosis, not widely available for screening, and can only predict preterm births up to seven days. In order to improve the prediction efficiency, some researchers changed the modeling method, QUiPP App v.2 (26), while some researchers added the prediction variables, placenta alpha microglobulin-1, phosphorylated insulin-like growth factor binding protein-1, and cervical length, respectively (27). However, serum samples were used from the second trimester to build the model in our study, which could predict preterm birth in earlier time. Besides, five variables were selected to enter the regression model to improve the prediction efficiency.

The limitation of this study is that the sample size we use is small. Furthermore, the prediction model was established by machine learning to expand samples to validate the efficiency of prediction. However, our nested case-control study is still ongoing, we will expand the sample size to further verify the prediction efficiency of the model. Moreover, we plan to verify the different expression level of cortisol in urine and saliva samples among the sPTB and the full-term group, which may provide an idea to further establish a non-invasive prediction model in the future.

\section{CONCLUSION}

In summary, validation of the prediction model suggests that high scores of Zung Self-Rating Depression Scale, high level of cortisol, Eicosane, methyltetradecanoate and lower level of stearic acid in maternal serum can reliably predict the risk of sPTB in asymptomatic pregnant women in the second trimester.

\section{DATA AVAILABILITY STATEMENT}

The raw data supporting the conclusions of this article will be made available by the authors, without undue reservation.

\section{ETHICS STATEMENT}

The studies involving human participants were reviewed and approved by The First Affiliated Hospital of Chongqing Medical University Ethics Committee. The patients/participants provided their written informed consent to participate in this study.

\section{AUTHOR CONTRIBUTIONS}

$\mathrm{DH}$ : conceptualization. $\mathrm{DH}$ and $\mathrm{ZL}$ : data curation and formal analysis. YB: resources. XLi and MW: software. XLu and HQ: supervision. All authors made substantial contributions to the paper and read and approved the final manuscript.

\section{FUNDING}

This work was supported by grants from the National Key R\&D Program of China (No.2016YFC1000407); the National Natural Science Foundation of China (No.81771614 and No.81771613); the Science and Technology Department of Sichuan Province (No.2020YFQ0006).

\section{ACKNOWLEDGMENTS}

We thank the participants in this prospective cohort study and the study physicians and nurses for their contributions to this study. 


\section{REFERENCES}

1. Chawanpaiboon S, Vogel JP, Moller A-B, Lumbiganon P, Petzold M, Hogan D, et al. Global, regional, and national estimates of levels of preterm birth in 2014: a systematic review and modelling analysis. Lancet Global Health. (2019) 7:e37-46. doi: 10.1016/S2214-109X(18)30451-0

2. Chen C, Zhang JW, Xia HW, Zhang HX, Betran AP, Zhang L, et al. Preterm birth in China between 2015 and 2016. Am J Public Health. (2019) 109:1597604. doi: 10.2105/AJPH.2019.305287

3. Frey HA, Klebanoff MA. The epidemiology, etiology, and costs of preterm birth. Semin Fetal Neonatal Med. (2016) 21:68-73. doi: 10.1016/j.siny.2015.12.011

4. Bodeau-Livinec F, Marlow N, Ancel P-Y, Kurinczuk JJ, Costeloe K, Kaminski M. Impact of intensive care practices on short-term and long-term outcomes for extremely preterm infants: comparison between the British Isles and France. Pediatrics. (2008) 122:e1014-21. doi: 10.1542/peds.2007-2976

5. Wu F, Liu G, Feng Z, Tan X, Yang C, Ye X, et al. Short-term outcomes of extremely preterm infants at discharge: a multicenter study from Guangdong province during 2008-2017. BMC Pediatr. (2019) 19:405. doi: 10.1186/s12887-019-1736-8

6. Staneva A, Bogossian F, Pritchard M, Wittkowski A. The effects of maternal depression, anxiety, and perceived stress during pregnancy on preterm birth: a systematic review. Women Birth. (2015) 28:179-93. doi: 10.1016/j.wombi.2015.02.003

7. Eick SM, Meeker JD, Swartzendruber A, Rios-McConnell R, Brown P, Vélez-Vega C, et al. Relationships between psychosocial factors during pregnancy and preterm birth in Puerto Rico. PLoS ONE. (2020) 15:e0227976. doi: 10.1371/journal.pone. 0227976

8. Hoffman MC, Mazzoni SE, Wagner BD, Laudenslager ML, Ross RG. Measures of maternal stress and mood in relation to preterm birth. Obstet Gynecol. (2016) 127:545-52. doi: 10.1097/AOG.0000000000001287

9. García-Blanco A, Diago V, Serrano De La Cruz V, Hervás D, CháferPericás C, Vento M. Can stress biomarkers predict preterm birth in women with threatened preterm labor? Psychoneuroendocrinology. (2017) 83:19-24. doi: 10.1016/j.psyneuen.2017.05.021

10. Li X, Yin M, Gu J, Hou Y, Tian F, Sun F. Metabolomic profiling of plasma samples from women with recurrent spontaneous abortion. Med Sci Monit. (2018) 24:4038-45. doi: 10.12659/MSM.907653

11. Souza RT, McKenzie EJ, Jones B, de Seymour JV, Thomas MM, Zarate E, et al. Trace biomarkers associated with spontaneous preterm birth from the maternal serum metabolome of asymptomatic nulliparous women parallel case-control studies from the SCOPE cohort. Sci Rep. (2019) 9:13701. doi: 10.1038/s41598-019-50252-7

12. Horgan R, Clancy O, Myers J, Baker P. An overview of proteomic and metabolomic technologies and their application to pregnancy research. BJOG Int $J$ Obstetr Gynaecol. (2009) 116:173-81. doi: 10.1111/j.1471-0528.2008.01997.x

13. Chi Y, Pei L, Chen G, Song X, Zhao A, Chen T, et al. Metabonomic profiling of human placentas reveals different metabolic patterns among subtypes of neural tube defects. J Proteome Res. (2014) 13:934-45. doi: 10.1021/pr4009805

14. Mathe EA, Patterson AD, Haznadar M, Manna SK, Krausz KW, Bowman $E D$, et al. Noninvasive urinary metabolomic profiling identifies diagnostic and prognostic markers in lung cancer. Cancer Res. (2014) 74:3259-70. doi: 10.1158/0008-5472.CAN-14-0109

15. Smart KF, Aggio RBM, Van Houtte JR, Villas-Bôas SG. Analytical platform for metabolome analysis of microbial cells using methyl chloroformate derivatization followed by gas chromatography-mass spectrometry. Nat Protoc. (2010) 5:1709-29. doi: 10.1038/nprot.2010.108

16. Xia J, Sinelnikov IV, Han B, Wishart DS. MetaboAnalyst 3.0-making metabolomics more meaningful. Nucleic Acids Res. (2015) 43:W251-7. doi: $10.1093 /$ nar/gkv380

17. Williams TC, Drake AJ. Preterm birth in evolutionary context: a predictive adaptive response? Phil Trans R Soc B. (2019) 374:20180121. doi: $10.1098 /$ rstb.2018.0121
18. Yonkers KA, Smith MV, Forray A, Epperson CN, Costello D, Lin H, et al. Pregnant women with posttraumatic stress disorder and risk of preterm birth. JAMA Psychiatry. (2014) 71:897-904. doi: 10.1001/jamapsychiatry.2 014.558

19. Owen DJ, Wood L, Tomenson B, Creed F, Neilson JP. Social stress predicts preterm birth in twin pregnancies. J Psychosom Obstetr Gynecol. (2017) 38:63-72. doi: 10.1080/0167482X.2016.1235146

20. Manetti L, Rossi G, Grasso L, Raffaelli V, Scattina I, Del Sarto S, et al. Usefulness of salivary cortisol in the diagnosis of hypercortisolism: comparison with serum and urinary cortisol. Eur J Endocrinol. (2013) 168:315-21. doi: 10.1530/EJE-12-0685

21. Bandoli G, Jelliffe-Pawlowski LL, Feuer SK, Liang L, Oltman SP, Paynter R, et al. Second trimester serum cortisol and preterm birth: an analysis by timing and subtype. J Perinatol. (2018) 38:973-81. doi: 10.1038/s41372-018-0128-5

22. Sarafidis K, Begou O, Deda O, Gika H, Agakidis C, Efstathiou N, Theodoridis G. Targeted urine metabolomics in preterm neonates with intraventricular hemorrhage. J Chromatogr B Analyt Technol Biomed Life Sci. (2019) 1104, 240-48. doi: 10.1016/j.jchromb.2018.11.024

23. Ahsan T, Chen J, Zhao X, Irfan M, Wu Y. Extraction and identification of bioactive compounds (eicosane and dibutyl phthalate) produced by Streptomyces strain KX852460 for the biological control of Rhizoctonia solani AG-3 strain KX852461 to control target spot disease in tobacco leaf. AMB Expr. (2017) 7:54. doi: 10.1186/s13568-017-0351-z

24. Wallace JL, Aland KL, Blatt K, Moore E, DeFranco EA. Modifying the risk of recurrent preterm birth: influence of trimester-specific changes in smoking behaviors. Am J Obstetr Gynecol. (2017) 216:310.e1-310.e8. doi: 10.1016/j.ajog.2016.11.1034

25. Cobo T, Aldecoa V, Figueras F, Herranz A, Ferrero S, Izquierdo M, et al. Development and validation of a multivariable prediction model of spontaneous preterm delivery and microbial invasion of the amniotic cavity in women with preterm labor. Am J Obstetr Gynecol. (2020) 223:421.e1-e14. doi: 10.1016/j.ajog.2020.02.049

26. Carter J, Seed PT, Watson HA, David AL, Sandall J, Shennan AH, et al. Development and validation of predictive models for QUiPP App v.2: tool for predicting preterm birth in women with symptoms of threatened preterm labor. Ultrasound Obstet Gynecol. (2020) 55:357-67. doi: 10.1002/uog.20422

27. Nikolova T, Uotila J, Nikolova N, Bolotskikh VM, Borisova VY, Di Renzo GC. Prediction of spontaneous preterm delivery in women presenting with premature labor: a comparison of placenta alpha microglobulin-1, phosphorylated insulin-like growth factor binding protein-1, and cervical length. Am J Obstetr Gynecol. (2018) 219:610.e1-e9. doi: 10.1016/j.ajog.2018.09.016

Conflict of Interest: The authors declare that the research was conducted in the absence of any commercial or financial relationships that could be construed as a potential conflict of interest.

The reviewer LW declared a shared affiliation, with no collaboration, with the authors to the handling editor at the time of the review.

Publisher's Note: All claims expressed in this article are solely those of the authors and do not necessarily represent those of their affiliated organizations, or those of the publisher, the editors and the reviewers. Any product that may be evaluated in this article, or claim that may be made by its manufacturer, is not guaranteed or endorsed by the publisher.

Copyright $\odot 2021$ Huang, Liu, Liu, Bai, Wu, Luo and Qi. This is an open-access article distributed under the terms of the Creative Commons Attribution License (CC $B Y)$. The use, distribution or reproduction in other forums is permitted, provided the original author(s) and the copyright owner(s) are credited and that the original publication in this journal is cited, in accordance with accepted academic practice. No use, distribution or reproduction is permitted which does not comply with these terms. 